\title{
PRELIMINARY ANALYSES OF PSYCHOMETRIC CHARACTERISTICS OF THE POLISH VERSION OF THE OBSESSIVE-COMPULSIVE INVENTORY-REVISED (OCI-R) IN A NON-CLINICAL SAMPLE
}

\section{JUSTYNA MOJSA-KAJA ${ }^{1,2}$, KRYSTYNA GOLONKA ${ }^{1}$, and MAGDA GAWŁOWSKA ${ }^{2}$}

Jagiellonian University, Kraków, Poland

${ }^{1}$ Institute of Applied Psychology, Department of Neurobiology and Neuropsychology

${ }^{2}$ Malopolska Centre of Biotechnology, Neurobiology Department

\begin{abstract}
Objectives: Obsessive-compulsive disorder (OCD) is an anxiety-spectrum disorder that affects $1-2 \%$ of the adult population. People with OCD are more likely to report impaired social and occupational functioning. Although effective treatments of the OCD exist, many sufferers from this disorder are continuously misdiagnosed. Therefore, improving the assessment of the OCD remains an important area of scientific research. The main goal of the study is the initial verification of psychometric properties in the Polish version of the Obsessive-Compulsive Inventory-Revised (OCI-R) in a college student sample. Material and Methods: A group of students completed a battery of measures consisting of obsessive-compulsive symptoms (The OCI-R, The Yale-Brown Obsessive Compulsive Scale), depression (The Beck Depression Inventory) and anxiety trait (The State-Trait Anxiety Inventory). Results: A confirmatory factor analysis, conducted on data from 334 university students, supported a solid and replicable 6-fold factor structure of the OCI-R. Further analyses on test-retest reliability (following a 1-month interval), convergent and divergent validity of the OCI-R were respectively conducted in a group of 137 students who had completed a battery of measures mentioned above. The results showed adequate testretest reliability for the full scale and subscales cores, high internal consistency and confirmed satisfactory convergent and divergent validity. Conclusions: The study constitutes the first phase of work on a Polish version of measurement for obsessive-compulsive symptoms. Satisfactory results obtained in a non-clinical sample allow to recognize this method to be promising for further research. Int J Occup Med Environ Health 2016;29(6):1011-1021.
\end{abstract}

Key words:

Assessment, Obsessive-compulsive disorder, OCI-R, Obsessive-compulsive symptoms, Non-clinical sample,

Obsessive-Compulsive Inventory-Revised

Funding: the research was supported by National Science Centre grant No. 2012/05/E/HS6/03553 entitled "Neural correlates of temperament dimensions in error monitoring and learning under reward and punishment conditions - dEEG and fMRI studies." Grant manager: Justyna Mojsa-Kaja, Ph.D.

Received: September 8, 2015. Accepted: January 13, 2016.

Corresponding author: J. Mojsa-Kaja, Jagiellonian University, Institute of Applied Psychology, Department of Neurobiology and Neuropsychology, Łojasiewicza 4, 30-348 Kraków, Poland (e-mail: justyna.mojsa-kaja@uj.edu.pl). 


\section{INTRODUCTION}

The obsessive-compulsive disorder (OCD) is an anxietyspectrum disorder characterized by persistently intrusive thoughts (e.g., obsessions) which generate an overbearing sense of fear or worry and spawn habitually repetitive actions (e.g., compulsions) as reactionary mechanisms aimed at reducing the associated anxiety. Obsessive-compulsive disorder severely affects $1-2 \%$ of the adult population and is associated with impairments in the social and occupational areas [1] but is nonetheless exhibited in 1 minor manifestation or another across the generalized population.

Prominent obsession and compulsion studies include Gibbs [2], Johansen and Dittrich [3], Novara et al. [4], Sulkowski et al. [5,6], Tolin [7]. Representative study data indicates that obsessive and compulsive symptoms manifest frequently in a manageable manner in the general population $[2,3]$ with varying degrees of possession and execution. Obsessive-compulsive tendencies in otherwise healthy individuals are analyzed in relation to anxiety, depression, thinking styles, and personality traits. Gibbs [2] have shown that scores of anxiety in non-clinical obsessive-compulsive samples lie somewhere between those possessing normal control subjects and the OCD-diagnosed patients.

Considerations involving etiological factors in the development of the OCD, including inflated responsibility and guilt, need for control, and exaggerated responsiveness to emotional provocation are described [2]. Obsessive-compulsive disorder symptoms may lead to impairments in individuals' functioning and hinder the ability to adequately perform daily tasks. Gibbs [2] showed that, compared to control subjects, non-clinical obsessive-compulsive sample patients performed at a significant disadvantage on the abilities of executive functioning, memory, and information processing. Although the Johansen and Dittrich [3] research findings have not confirmed the significant impact of the OCD symptoms on cognitive impairments, they emphasized that the symptoms may have diminish the quality of life as well as diminished influenced the occupational activity. Mancebo et al. [8] found that $1 / 3$ of the studied sample reported an inability to work due to psychopathology and $14 \%$ reported receiving disability benefits because of their OCD symptoms. Abramowitz et al. [9] noted that people with the OCD were more likely to be unemployed, and more likely to report impaired social and occupational functioning, compared with those diagnosed with anxiety or unipolar mood disorders.

Although effective treatments of the OCD exist, many sufferers from this disorder are continuously misdiagnosed. Therefore, improving the assessment of the OCD remains an important area of scientific research and practical application in both clinical and non-clinical domains. There are many types of instruments used in the assessment and diagnosis of the OCD [10,11]. One of these instruments is the Obsessive-Compulsive Inventory-Revised (OCI-R) [12], which is a self-reported measure consisting of a total of 18 items that provide scores on 6 subscales of 3 items each (allowing for comparisons) which are summed up as: washing, checking, ordering, obsessing, hoarding, and neutralizing. Due to clear distinction of 6 subscales, the inventory may be used to identify homogenous OCD patient groups for research on the pathology of specific symptom dimensions that could expand our understanding of particular aspects of the OCD and analyses of their sensitivity to certain treatment.

It has overcome the limitations of previously developed methods, stemming mainly from insufficient coverage of the diversity of symptoms [13]. The heterogeneity of obsessive and compulsive symptoms have required validation of the instrument that measures the wide range of empirically driven symptom presentations. The OCI-R address this aim, assessing distress linked with major common concerns of patients with the OCD and is an advantage over other OCD measures [10,11]. Additionally, due to its brevity and simplicity it is an example of an instrument 
that has potential for a very practical use in screening in non-clinical samples and as well as in research in clinical settings.

The original English version has excellent psychometric properties both in a sample of patients with the OCD, other anxiety disorders and non-clinical populations such as college students [12,14]. Therefore, OCI-R seems to be a reliable measure for the assessment of the OCD in different samples and a promising method for screening and conducting research in large populations.

Although the occurrence rate of the OCD remains stable regardless of culture taken into consideration, the replication of any research across different societies requires the use of a method that has been properly and culturally adapted. As of today, in Poland, there is only one official method - The Yale-Brown Obsessive Compulsive Scale (Y-BOCS) focused on diagnosis of the OCD among adults, which has been psychometrically verified [15]. As a result, the main goal of this study is the preliminary verification of psychometric properties of the Polish version of the OCI-R in a college student sample including the initial examination of its reliability, factor structure, and both its divergent and convergent validity.

\section{MATERIAL AND METHODS}

\section{Participants and procedure}

The OCI-R study was divided into 2 stages. In stage 1, a sample of 334 students (male/female ratio $=71 / 263$, mean age: $20.07 \pm 1.85$ years old) from the Polish university (Jagiellonian University, Kraków) completed only the OCI-R. The data in stage 1 was used primarily to verify 3 main goals: a factorial structure, correlations among OCI-R scales and internal consistency.

The 2nd stage of the study was conducted 4 weeks following the stage 1 and included a group of 137 student participants (male/female $=30 / 107$, mean age: $19.46 \pm 1.06$ years old) from the stage 1 of the study. All participants were administered the questionnaires in a group setting during a lecture period. A brief description of the purpose of the study was given and then students were asked to complete a battery of measures assessing obsessive-compulsive symptoms, depression and anxiety trait and constituted the final sample for test-retest reliability, convergent and divergent validity. Participation was voluntary, anonymous and no payment or course credits were offered to the participants.

Since Hajcak et al. [14] found significant order effects for the OCI-R and the Maudsley Obsessive-Compulsive Inventory (MOCI), with a finding of lower means when each measure was presented 2 nd in line, 71 student participants completed the OCI-R first, followed by their completion of the Y-BOCS and other measures and 66 of the student participants initially answered Y-BOCS, followed by answering the OCI-R and other measures they participated in.

\section{Measures}

The Obsessive-Compulsive Inventory-Revised (OCI-R)

The OCI-R [12] is a self-reporting measure to assess the distress associated with obsessions and compulsions in both clinical and non-clinical samples. Participants rate the degree to which they were bothered or distressed by the OCD symptoms in the past month on a 5-point scale from 0 (not at all) to 4 (extremely). The OCI-R assesses the OCD symptoms across 6 factors: 1) washing, 2) checking, 3) obsessing, 4) neutralizing, 5) ordering, and 6) hoarding. The OCI-R consists of 18 items equally divided into those 6 subscales.

Both the English and other originally translated OCI-R language versions have proven satisfactory psychometric properties [12-14,16].

The original version of this measure was translated into Polish and then back-translated into English in order to verify the accuracy of the translation. More precisely, after having received authorization from the authors 
of the instrument, it was translated and adapted to Polish. The translation from English into Polish was done independently by 3 bilingual psychologists, the authors of the paper. Then, these 3 translations were compared and merged, resulting in an initial Polish version. Once the inventory in Polish had been considered adequate for use, it was independently back-translated into English by a professional bilingual translator whose version was sent to the authors of the original method for acceptance. During the process of cross-cultural adaptation of the OCI-R, the authors focused on obtaining high correspondence between the original method and translated version.

To assess the validity of the Polish version of the OCI-R three additional instruments were chosen - for The YaleBrown Obsessive Compulsive Scale for convergent validity and The Beck Depression Inventory and The StateTrait Anxiety Inventory for divergent validity. The choice of these methods was driven by restricted availability of the OCD measures in Polish (as of today only Y-BOCS focused on diagnosis of OCD among adults have been psychometrically verified) and an attempt to compare research protocol with other language version already published [e.g., 12,13].

The Yale-Brown Obsessive Compulsive Scale (Y-BOCS)

The Yale-Brown Obsessive Compulsive Scale [17] was designed to assess the severity of the obsessive-compulsive disorder independently from the variety of obsessions and compulsions present. It explores 5 varying dimensions for obsessions as well as 5 for compulsions. Each item is rated 0-4 and includes both item probes and anchor points to guide the surveyed. The Polish version of the Y-BOCS [18] exhibited good psychometric properties [15].

\section{The Beck Depression Inventory (BDI)}

The Polish version [19] of the BDI [20], is a 21-item selfreport scale employed for assessing both the cognitive and physical symptoms of depression, which have both been frequently utilized in psychological research due to their reliability and validity [21].

The State-Trait Anxiety Inventory, Trait subscale (STAI-T)

The Polish version [22] of the STAI-T [23] determined trait anxiety. The STAI-T is a 20 -item questionnaire most frequently used for measuring of trait anxiety in psychological research studies due to its psychometric properties.

\section{RESULTS}

The confirmatory factor analysis was conducted using AMOS version 22, while the remaining statistical analyses were performed using SPSS version 21 (IBM SPSS Statistics, IBM Corporation, USA).

\section{Factor structure of the Polish OCI-R}

This confirmatory factor analysis was performed in order to evaluate whether this data fitted the original 6-factor structure proposed by Foa et al. [12]. To facilitate comparability, we used the same fit indices as Foa et al. [12] and Hajcak et al. [14] did. An ideal model fitness may be indicated by a nonsignificant item-trait interaction $\mathrm{Chi}^{2}$ probability value test. However, because the $\mathrm{Chi}^{2}$ test had been criticized for its dependence on sample size, a range of fit statistics was assessed. A relative $\mathrm{Chi}^{2}$ is usually applied (the ratio of $\mathrm{Chi}^{2}$ to degrees of freedom - $\mathrm{Chi}^{2} / \mathrm{df}$ ) with a recommended range 1-2 [24]. The root mean square error approximation (RMSEA) values between 0.05-0.08 indicate a reasonable fit and the standardized root mean square residual (SRMR) values $<0.08$ indicate an ideal fit. Some authors prefer to use the root mean square residual (RMR) which calls for sub-0.06. For the fit statistics (via comparative fit index (CFI)), sub-0.90, in accordance with the goodness-of-fit index (GFI), indicates a lack of reasonable fit, while 0.9-0.95 indicates a reasonable fit. Furthermore, values of $>0.95$ indicate an optimum fit [25]. 
Three hundred and thirty-four participants were involved in the 1st part of the study. Therefore, the ratio participants/items was 18.55/1 and was a significantly superior ratio to the recommended minimum subject-toitem ratio of 5/1 [26]. The confirmatory factor analysis with the maximum-likelihood estimation was conducted to evaluate the model adequacy with all the fit indices listed above. Latent factors were allowed to vary, while errors were not. The model had a significant $\mathrm{Chi}^{2}$ $\left(\mathrm{Chi}^{2}(120)=223.796, \mathrm{p}<0.000\right), \mathrm{Chi}^{2} / \mathrm{df}=1.865$ within the recommended range of 1 to 2, a GFI of 0.93 , a CFI of 0.95 , a SRMR of 0.05 , a RMR of 0.06 , and RMSEA of 0.05 .

Considering criteria by $\mathrm{Hu}$ and Bentler [25], our results indicate a good fit for the 6-factor model, with similar values presented by Foa et al. [12] and Hajcak et al. [14] in the English original version and Fullana et al. in the Spanish version [13].

A 1-factor model was also tested for the 18 items, and all indices suggested poor fitness as compared to the 6 -factor model $\left(\mathrm{Chi}^{2}(135)=1005.289, \mathrm{p}<0.000\right), \mathrm{Chi}^{2} / \mathrm{df}=7.447$, GFI of 0.73 , a CFI of 0.55 , a SRMR of 0.1 , a RMR of 0.13 and RMSEA of 0.14 .

\section{Correlation among OCI-R scales}

The criteria of correlation size evaluation, as suggested by Cohen [27], were utilized. Correlations $>0.5$ were defined as "large," correlations ranging from 0.3-0.49 were defined as "medium," and correlations $0.1-0.29$ were deemed "small." Although all correlations of the OCI-R between the subscale and total scale were "large," the intercorrelations among the subscales were determined to be "small" and "medium" correlations, ranging $0.2-0.4$, and proving a small overlap to exist among them (Table 1).

Descriptive statistics, gender differences and internal consistency. The Table 2 presents the means, standard subscale deviations, and total OCI-R. The mean OCI-R total score in this study was slightly higher than in previous studies with similar samples [13,14]. Women score higher than men in the OCI-R $(\mathrm{t}(325)=2.3, \mathrm{p}=0.02)$ overall, and additionally attain a higher score on the wash$\operatorname{ing}(\mathrm{t}(325)=2.14, \mathrm{p}=0.03)$ and obsessing $(\mathrm{t}(325)=2.18$, $p=0.03)$ aspects, respectively.

Cronbach's $\alpha$ was calculated to assess the internal consistency of the OCI-R total scale, as well as each of the subscales, as compared with results of previous studies with similar samples (Table 3). While the OCI-R total scale, obsessing subscale demonstrate good internal consistency, washing, ordering and checking aspects show acceptable internal consistency, the hoarding and neutralizing aspects demonstrate moderate internal consistency. The lowest alpha values for neutralizing and hoarding are consistent with other studies (see Table 3).

Table 1. Correlations among the Obsessive-Compulsive Inventory-Revised (OCI-R) subscales*

\begin{tabular}{lccccccc}
\hline \multirow{2}{*}{ Subscale } & \multicolumn{7}{c}{ Correlations } \\
\cline { 2 - 8 } & 1 & 2 & 3 & 4 & 5 & 6 & total \\
\hline 1. Washing & - & 0.29 & 0.23 & 0.31 & 0.35 & 0.33 & 0.62 \\
2. Obsessing & & - & 0.37 & 0.20 & 0.39 & 0.29 & 0.68 \\
3. Hoarding & & & - & 0.24 & 0.40 & 0.33 & 0.65 \\
4. Ordering & & & - & 0.39 & 0.30 & 0.62 \\
5. Checking & & & & - & 0.34 & 0.74 \\
6. Neutralizing & & & & & - & 0.62 \\
\hline
\end{tabular}

* All p-values $<0.05$. 
Table 2. Obsessive-Compulsive Inventory-Revised (OCI-R) scores by gender

\begin{tabular}{|c|c|c|c|c|}
\hline & \multirow[t]{2}{*}{ OCI-R } & \multicolumn{3}{|c|}{$\begin{array}{c}\text { Respondents' scores* } \\
(\mathrm{M} \pm \mathrm{SD})\end{array}$} \\
\hline & & males & females & total \\
\hline Total & & $19.69 \pm 11.65$ & $22.94 \pm 10.20$ & $22.23 \pm 10.60$ \\
\hline \multicolumn{5}{|l|}{ Subscale } \\
\hline washing & & $1.61 \pm 1.98$ & $2.29 \pm 2.46$ & $2.14 \pm 2.38$ \\
\hline obsessing & & $4.59 \pm 3.39$ & $5.54 \pm 3.17$ & $5.33 \pm 3.24$ \\
\hline hoarding & & $4.08 \pm 2.75$ & $4.53 \pm 2.51$ & $4.44 \pm 2.57$ \\
\hline ordering & & $4.28 \pm 2.96$ & $4.80 \pm 2.74$ & $4.71 \pm 2.81$ \\
\hline checking & & $3.39 \pm 2.98$ & $3.97 \pm 2.79$ & $3.85 \pm 2.85$ \\
\hline neutralizing & & $1.73 \pm 2.14$ & $1.80 \pm 2.26$ & $1.77 \pm 2.22$ \\
\hline
\end{tabular}

* Based on a 5-point Likert's scale.

$\mathrm{M}$ - mean; SD - standard deviation.

Table 3. Obsessive-Compulsive Inventory-Revised (OCI-R) internal consistency

\begin{tabular}{lcccc}
\hline \multirow{2}{*}{ OCI-R } & \multicolumn{4}{c}{ Cronbach's $\alpha$} \\
\cline { 2 - 5 } & $\begin{array}{c}\text { English OCI-R version } \\
\text { (Hajcak et al. [14], 2004) }\end{array}$ & $\begin{array}{c}\text { Spanish OCI-R version } \\
\text { (Fullana et al. [13], 2005) }\end{array}$ & $\begin{array}{c}\text { French OCI-R version } \\
\text { (Zermatten et al. [16], 2006) }\end{array}$ & $\begin{array}{c}\text { Polish OCI-R version } \\
\text { (current-study) }\end{array}$ \\
\hline Total & 0.88 & 0.86 & 0.86 & 0.85 \\
Subscale & & & & \\
washing & 0.76 & 0.63 & 0.70 & 0.73 \\
obsessing & 0.77 & 0.82 & 0.78 & 0.85 \\
hoarding & 0.68 & 0.69 & 0.70 & 0.66 \\
ordering & 0.84 & 0.67 & 0.79 & 0.77 \\
checking & 0.76 & 0.77 & 0.83 & 0.74 \\
neutralizing & 0.61 & 0.61 & 0.63 & 0.62 \\
\hline
\end{tabular}

Test-retest reliability

Pearson's $r$ was calculated to assess test-retest reliability. Results of the test-retest reliability, after a 1-month interval for the OCI-R's total score and its subscales, are similar to those obtained in previously conducted research (Table 4). All coefficients indicate a moderate-to-excellent test-retest reliability rating, ranging $0.6-0.82$.

\section{Convergent validity for the $\mathrm{OCI}-\mathrm{R}$}

Convergent OCI-R total scale validation was verified by correlating the OCI-R with Y-BOCS, another measure of obsessive-compulsive symptoms targeting adult participants, which had been psychometrically verified in Poland [15]. The Y-BOCS total score correlated 0.57 with the OCI-R total, indicating moderate convergent validity (as presented in the Table 5).

\section{Order effects}

Similarly to Hajcak et al. [14] we found an order of effects on repeated administration of the OCI-R, despite the fact that this tendency was reversed. Means were nonetheless significantly higher when the OCI-R was 
Table 4. Obsessive-Compulsive Inventory-Revised (OCI-R) test-retest reliability (1 month)

\begin{tabular}{lcccc}
\hline \multirow{2}{*}{ OCI-R } & \multicolumn{4}{c}{ Reliability } \\
\cline { 2 - 5 } & $\begin{array}{c}\text { English OCI-R version } \\
\text { (Foa et al. [12], 2002 } \\
\text { control group) }\end{array}$ & $\begin{array}{c}\text { English OCI-R version } \\
\text { (Hajcak et al. [14], 2004) }\end{array}$ & $\begin{array}{c}\text { Spanish OCI-R version } \\
\text { (Fullana et al. [13], 2005) }\end{array}$ & $\begin{array}{c}\text { Polish OCI-R version } \\
\text { (current-study) }\end{array}$ \\
\hline Total & 0.84 & 0.70 & 0.67 & 0.74 \\
Subscale & & & & \\
washing & 0.87 & 0.72 & 0.45 & 0.82 \\
obsessing & 0.66 & 0.77 & 0.57 & 0.73 \\
hoarding & 0.78 & 0.58 & 0.56 & 0.74 \\
ordering & 0.84 & 0.61 & 0.66 & 0.65 \\
checking & 0.75 & 0.75 & 0.68 & 0.68 \\
neutralizing & 0.57 & 0.54 & 0.50 & 0.60 \\
\hline
\end{tabular}

Table 5. Convergent validity for the Obsessive-Compulsive Inventory-Revised (OCI-R)*

\begin{tabular}{|c|c|}
\hline & $\begin{array}{l}\text { OCI-R score } \\
\text { (total) }\end{array}$ \\
\hline Total & 0.57 \\
\hline \multicolumn{2}{|l|}{ Subscale } \\
\hline obsessions & 0.54 \\
\hline compulsions & 0.48 \\
\hline
\end{tabular}

* All p-values $<0.05$.

Y-BOCS - The Yale-Brown Obsessive Compulsive Scale.

administered before $($ mean $(M)=20.38$, standard deviation $(\mathrm{SD})=10.7)$ vs. after $(\mathrm{M}=13.45, \mathrm{SD}=8.03)$ the Y-BOCS $(\mathrm{t}(135)=4.26, \mathrm{p}=0.001)$. There were no order effects in the case of Y-BOCS $(\mathrm{t}(135)=-1.93, \mathrm{p}=0.06)$.

\section{Divergent validity for the $\mathrm{OCl}-\mathrm{R}$}

To assess the divergent in validity, the correlation between the OCI-R, BDI and STAI-T scores were calculated (see the Table 6).

Although the correlation between OCI-R total score, BDI and STAI-T was in most cases statistically significant, they were only moderate (Table 6) and lower than that of the Y-BOCS total score (Table 5).
Table 6. Divergent validity for the Obsessive-Compulsive Inventory-Revised (OCI-R)*

\begin{tabular}{lll}
\hline \multirow{2}{*}{ OCI-R } & \multicolumn{2}{c}{ Divergent validity } \\
\cline { 2 - 3 } Total & BDI & STAI-T \\
Subscale & 0.34 & 0.32 \\
washing & & \\
obsessing & 0.28 & 0.15 \\
hoarding & 0.50 & 0.45 \\
ordering & 0.14 & 0.21 \\
checking & 0.04 (n.s.) & 0.12 (n.s.) \\
neutralizing & 0.19 & 0.18 \\
\hline
\end{tabular}

* All p-values $<0.05$, except where indicated as n.s. - not statisticaly significant.

BDI - The Beck Depression Inventory; STAI-T - The State-Trait Anxiety Inventory, Trait Subscale.

\section{DISCUSSION}

This study demonstrates psychometric properties of a Polish version of the OCI-R in a non-clinical sample. It proves to have a solid and replicable 6-factor structure, comparable with that previously received in both clinical [12] and non-clinical samples $[13,14]$. These results postulate that the OCD might be more accurately defined as groups of syndromes rather than as an unidimensional construct. 
This postulation is in line with the current research on the OCD, which suggests that each respective symptomatic dimension is perhaps associated with varying etiology and thus requires specific treatment [28].

Referencing internal reliability, the Polish version of the OCI-R shows Cronbach's $\alpha$ values similar to previous studies $[13,14,16]$ suggesting good reliability. Because of the weaker internal consistency of the 2 scales of hoarding and neutralizing, further studies on internal consistency of those groups of symptoms would be required.

The OCI-R total and subscale scores demonstrated in the current study show promising test-retest reliability spanned over 4 weeks and are comparable with those reported by other authors [12-14,16].

Convergent validity of a Polish version of OCI-R was moderate with Y-BOCS, which was used as a self-report measure in this study. Although Y-BOCS is a semi-structured interview that primarily evaluates symptom severity and treatment responses of people with the OCD, it has been previously used as a self-reporting measure in a non-clinical sample [29-31]. Self-reporting versions of Y-BOCS tested in non-clinical samples confirm the originally proposed structure while exhibiting both satisfactory internal consistency and convergent validity [29]. Furthermore, it is described as both a reliable and a valid instrument for the assessment of the severity of obsessions and compulsions in a student population [30]. Although, the self-report version of Y-BOCS is widely used in the healthy population, some authors suggest a caution in the use of Y-BOCS administered in this manner in clinical samples [31,32].

Due to the lack of alternative psychometrically-verified methods for measuring the OCD among adults used in Polish language studies, the further feasible examination with other measures proved impossible at this stage of research. In terms of divergent validity, the correlation between OCI-R total score and both BDI and STAI-T were small suggesting adequate discriminative validity. Similar to previous studies, the highest recorded correlations were between obsessing and both BDI [14] and STAI-T scores [13] thus suggesting some overlap between this group of the OCD symptoms, depression and trait anxiety. Nevertheless, those correlations were lower than that with the Y-BOCS total score. Therefore, despite the fact that some overlapping exists between depression, trait anxiety and obsessive-compulsive symptoms, the OCI-R proves to measure a distinct construct.

In line with Hajcak et al. [14] we found order effects on repeated administration of the OCI-R. However, this tendency was reversed (due to the means being significantly higher when the OCI-R was given before vs. after the Y-BOCS). In contrast to order effects noted by Hajcak et al. [14] in the case of MOCI, there were no order effects in any case of Y-BOCS. On the other hand, Fullana et al. [13] did not confirm such effects in cases of OCI-R measured with Padua Inventory. Nevertheless, further studies employing OCI-R should be wary of questionnaire order effects.

Finally, according to analyzed sex differences on the basis of the OCI-R scores, women scored higher than men in the OCI-R's total score and on washing and obsessing subscales. Fullana et al. [13] on the other hand found that men scored higher than women on the hoarding and checking scales.

It must be noted that there is the lack of result consistency of existing research on the OCD symptoms analyzed from the perspective of sex differences. These incongruences may stem from the focus of different methods based on the severity or frequency of these symptoms.

Taking into account the scales of the OCD, it is crucial to analyze and describe this problem in the general population. This may develop the awareness of the existence on non-clinical obsessive-compulsive sample [2] or in other terms such as subclinical OCD [3] in the healthy population. Being aware of the symptoms' severity and that their influence on one's performance and productivity 
may induce an individual to seek the treatment and support. The development of reliable and verified tools that simply scan subjects from non-clinical population would likely lead to quicker, more appropriate diagnoses and adequately effective therapies. In this context, the presentation in a globally recognized dialect version of this OCD measure stands to be a significant and invaluable contribution towards the development of further research on anxiety disorders. The availability of that instrument in Polish will certainly provide the researchers with additional tools to assess the severity of obsessive-compulsive symptoms.

\section{Limitations}

The current study has some important limitations that should be noted. Firstly, the presented study was conducted on non-clinical, student population. In future it is necessary to include an independent evaluation of OCI-R in clinical settings and further determine optimal cut scores which yield the optimal combination of sensitivity (identifying true positives) and specificity (identifying true negatives). Moreover, the information regarding the OCI-R's discriminative ability should be obtained. Secondly, all the data was based on self-reports. In the future, additional and more objective methods of obsessive-compulsive symptoms should be implemented in research procedure, such as standardized (clinical) interview or neurophysiological measures, as the methodology of electroencephalography (EEG) and functional magnetic resonance imaging (fMRI) research constantly sheds new light on the predetermined diagnostic criteria in various disorders.

\section{CONCLUSIONS}

In summary, the OCI-R is a brief and practical instrument for use in screening and research in large populations. The initial research on the Polish version provides that it is a sound psychometric measure of the OCD symptoms and shows similar psychometric indices to both the original and other language versions of OCI-R used in non-clinical samples. The results confirmed the 6-factor structure and showed adequate test-retest reliability for the full scale and subscale cores, high internal consistency, and satisfactory convergent and divergent validity.

This study constitutes the 1st phase of work on a Polish version of measurement for obsessive-compulsive symptoms. Satisfactory results obtained in a non-clinical sample, allow to recognize this method to be promising for further research. Currently, it requires verification on a clinical sample. Further work on the presented method may develop research in the area of obsessive-compulsive disorder, improve diagnosis and raise awareness on the impact of the obsessive-compulsive symptoms on individuals' functioning - both in clinical, and non-clinical samples.

\section{REFERENCES}

1. McGuire JF, Lewin AB, Horng B, Murphy TK, Storch EA. The nature, assessment, and treatment of obsessive-compulsive disorder. Postgrad Med. 2012;124(1):152-65, http:// dx.doi.org/10.3810/pgm.2012.01.2528.

2. Gibbs NA. Nonclinical populations in research on obsessivecompulsive disorder: A critical review. Clin Psychol Rev. 1996;16(8):729-73, http://dx.doi.org/10.1016/S0272-7358(96) 00043-8.

3. Johansen T, Dittrich WH. Cognitive performance in a subclinical obsessive-compulsive sample 1: Cognitive functions. Psychiatry J. 2013;2013:565191, http://dx.doi.org/10.1155/2013/ 565191.

4. Novara C, Pastore M, Ghisi M, Sica C, Sanavio E, McKay D. Longitudinal aspects of obsessive compulsive cognitions in a non-clinical sample: A five-year follow-up study. J Behav Ther Exp Psychiatry. 2011;42(3):317-24, http://dx.doi.org/ 10.1016/j.jbtep.2011.01.007.

5. Sulkowski ML, Jordan C, Reid A, Graziano PA, Shalev I, Storch EA. Relations between impulsivity, anxiety, and obsessive-compulsive symptoms in a non-clinical sample. Pers Individ Dif. 2009;47(6):620-5, http://dx.doi.org/10.1016/ j.paid.2009.05.020. 
6. Sulkowski ML, Mancil TL, Jordan C, Reid A, Chakoff E, Storch EA. Validation of a classification system of obsessivecompulsive spectrum disorder symptoms in a non-clinical sample. Psychiatry Res. 2011;188(1):65-70, http://dx.doi.org/ 10.1016/j.psychres.2011.01.015.

7. Tolin DF, Woods CM, Abramowitz JS. Disgust sensitivity and obsessive-compulsive symptoms in a non-clinical sample. J Behav Ther Exp Psychiatry. 2006;37(1):30-40, http:// dx.doi.org/10.1016/j.jbtep.2005.09.003.

8. Mancebo MC, Greenberg B, Grant JE, Pinto A, Eisen JL, Dyck I, et al. Correlates of occupational disability in a clinical sample of obsessive compulsive disorder. Compr Psychiatry. 2008;49(1):43-50, http://dx.doi.org/10.1016/j.comppsych. 2007.05.016.

9. Abramowitz JS, Taylor S, McKay D. Obsessive-compulsive disorder. Lancet. 2009;374(9688):491-9, http://dx.doi.org/ 10.1016/S0140-6736(09)60240-3.

10. Grabill K, Merlo L, Duke D, Harford KL, Keeley ML, Geffken GR, et al. Assessment of obsessive-compulsive disorder: A review. J Anxiety Disord. 2008;22(1):1-17, http:// dx.doi.org/10.1016/j.janxdis.2007.01.012.

11. Storch EA, Benito K, Goodman W. Assessment scales for obsessive-compulsive disorder. Neuropsychiatry. 2011; 1(3):243-50, http://dx.doi.org/10.2217/npy.11.22.

12. Foa EB, Huppert JD, Leiberg S, Langner R, Kichic R, Hajcak G, et al. The Obsessive-Compulsive Inventory: Development and validation of a short version. Psychol Assess. 2002;14(4):485-96, http://dx.doi.org/10.1037/10403590.14.4.485.

13. Fullana MA, Tortella-Feliu M, Caseras X, Andión Ó, Torrubia R, Mataix-Cols D. Psychometric properties of the Spanish version of the Obsessive-Compulsive Inventory - Revised in a non-clinical sample. J Anxiety Disord. 2005;19(8):893-903, http://dx.doi.org/10.1016/j.janxdis.2004.10.004.

14. Hajcak G, Huppert JD, Simons RF, Foa EB. Psychometric properties of the OCI-R in a college sample. Behav Res Ther. 2004;42(1):115-23, http://dx.doi.org/10.1016/j.brat. 2003.08.002.
15. Kiejna A, Grzesiak M, Małyszczak K. [The Yale-Brown Scale - A measure of the severity of obsessive-compulsive disorder]. Psychiatr Pol. 1999;32(1):69-76. Polish.

16. Zermatten A, van der Linden M, Jermann F, Ceschi G. Validation of a French version of the Obsessive-Compulsive Inventory-Revised in a non-clinical sample. Eur Rev Appl Psychol. 2006;56(3):151-5, http://dx.doi.org/10.1016/ j.erap.2005.07.003.

17. Goodman WK, Price LH, Rasmussen SA, Mazure C, Fleischmann RL, Hill CL, et al. The Yale-Brown Obsessive Compulsive Scale. I. Development, use, and reliability. Arch Gen Psychiatry. 1989;46(11):1006-11, http://dx.doi.org/10.1001/ archpsyc.1989.01810110048007.

18. Kiejna A, Grzesiak M. [The Yale-Brown Obsessive-Compulsive Scale (Y-BOCS) - A measure of the severity of obsessive-compulsive disorder]. Postepy Psychiatr Neurol. 1997;6:309-24. Polish.

19. Parnowski T, Jernajczyk W. [The Beck Depression Inventory in assessing mood in healthy subjects and patients with affective disorders]. Psychiatr Pol. 1977;11(4):417-21. Polish.

20. Beck AT, Ward CH, Mendelson M, Mock J, Erbaugh J. An inventory for measuring depression. Arch Gen Psychiatry. 1961;4:561-71, http://dx.doi.org/10.1001/archpsyc. 1961.01710120031004.

21. Beck AT, Steer RA, Carbin MG. Psychometric properties of the Beck Depression Inventory: Twenty-five years of evaluation. Clin Psychol Rev. 1988;8(1):77-100, http://dx.doi. org/10.1016/0272-7358(88)90050-5.

22. Spielberger CD, Strelau J, Tysarczyk M, Wrześniewski K. [The State-Trait Anxiety Inventory]. Warszawa: Pracownia Testow Psychologicznych; 1987. Polish.

23. Spielberger C, Gorsuch A, Lushene R. Manual for The State-Trait Anxiety Inventory. Palo Alto (CA): Consulting Psychologists Press; 1970.

24. Hair JF, Black WC, Babin BJ, Anderson RE, Tatham RL. Multivariate data analysis. 6th ed. Englewood Cliffs (NJ): Prentice Hall; 2005. 
25. Hu L, Bentler PM. Cutoff criteria for fit indexes in covariance structure analysis: Conventional criteria versus new alternatives. Struct Equ Modeling. 1999;6(1):1-55, http:// dx.doi.org/10.1080/10705519909540118.

26. MacCallum RC, Widaman KF, Zhang S, Hong S. Sample size in factor analysis. Psychol Methods. 1999;4(1):84-99, http://dx.doi.org/10.1037/1082-989X.4.1.84.

27. Cohen J. Statistical power analyses for the behavioral sciences. 2nd ed. Hillsdale (NJ): Erlbaum; 1988.

28. Benito K, Storch EA. Assessment of obsessive-compulsive disorder: Review and future directions. Expert Rev Neurother. 2011;11(2):287-98, http://dx.doi.org/10.1586/ ern.10.195.

29. Seol SH, Kwon JS, Shin MS. Korean self-report version of the Yale-Brown Obsessive-Compulsive Scale: Factor structure, reliability, and validity. Psychiatry Investig. 2013;10(1):17-25, http://dx.doi.org/10.4306/pi.2013.10.1.17.
30. Ólafsson RP, Snorrason Í, Smári J. Yale-Brown Obsessive Compulsive Scale: Psychometric properties of the selfreport version in a student sample. J Psychopathol Behav Assess. 2010;32(2):226-35, http://dx.doi.org/10.1007/s10862009-9146-0.

31. Wu KD, Watson D, Clark LA. A self-report version of the Yale-Brown Obsessive-Compulsive Scale Symptom Checklist: Psychometric properties of factor-based scales in 3 samples. J Anxiety Disord. 2007;21(5):644-61, http://dx.doi.org/ 10.1016/j.janxdis.2006.10.003.

32. Federici A, Summerfeldt LJ, Harrington JL, McCabe RE, Purdon CL, Rowa K, et al. Consistency between self-report and clinician-administered versions of the Yale-Brown Obsessive-Compulsive Scale. J Anxiety Disord. 2010;24(7): 729-33, http://dx.doi.org/10.1016/j.janxdis.2010.05.005.

This work is available in Open Access model and licensed under a Creative Commons Attribution-NonCommercial 3.0 Poland License - http://creativecommons.org/ licenses/by-nc/3.0/pl/deed.en. 